\title{
Abrigos Sensíveis, do método ao conceito, superando a instrumentalização
}

\author{
Sensitive Shelters: From methods to concepts, overcoming simple tooling approach \\ Andrés Passaro \\ PROURB FAU UFRJ, Brasil \\ andrespassaro@fau.ufrj.br \\ - Gonçalo Castro Henriques \\ FAU UFRJ, Brasil \\ gch@ufrj.br
}

\begin{abstract}
The following text reports a workshop activity performed at LAMO3d - Laboratório de Modelos 3d e Fabricação Digital, PROURB FAU UFRJ that addresses the instrumental domain within the new digital manufacturing capabilities such as 3D printing, laser cutting and parametric design, and on the other tries to overcome the activity as a mere technical training. This concern implies a larger effort that goes towards the incorporation of a coherent discourse and has the ability to validate within the contemporary setting these new operative actions.
\end{abstract}

Keywords: Digital Fabrication, Parametric Design, Responsive Architecture, Sensitive Shelters (Abrigos Sensíveis)

\section{Introdução}

O trabalho é um relato de uma atividade de Oficina, que tenta suprir a carência no domínio das novas tecnologias de Fabricação Digital, e que também aborda uma estratégia de ensino que supera os mecanismos de instrumentalização e desenvolve produtivamente, dentro de uma nova linguagem, uma nova ação operativa.

Uma ação operativa principalmente porque está ligada diretamente à produção do objeto. As poderosas ferramentas, a nível de simulação, permitem ter um entendimento efetivo do processo de produção do objeto arquitetônico. Entretanto não se trata de ensinar software, nem ferramentas de corte digital ou impressão 3d. Superar o discurso de técnica pela técnica implica na procura de novas linguagens junto às possibilidades que nos oferecem estes novos instrumentos.

A inovação tecnológica em desenho paramétrico aliada aos processos de fabricação digital tornam-se poderosos instrumentos com capacidade de modificar o discurso arquitetônico da atualidade. $\mathrm{O}$ entendimento deste novo ferramental é capaz de modificar processos de representação, modificar a utilização de materiais, modificar o processo produtivo e finalmente modificar o entendimento da forma arquitetônica. Estes condimentos colocam em crise os sistemas atuais da crítica e nos oferecem a receita para buscar e definir uma nova linguagem arquitetônica.
Sem dúvida que é possível utilizar os novos instrumentos para dar continuidade à produção arquitetônica atual que ainda oscila entre a fragmentação narrativa e a compacidade supermoderna (Moneo 1999). Também podem tornar-se grandes instrumentos para a construção da cidade do mercado. Porém, entendemos que pensar em continuidade é um retrocesso e que estas novas possibilidades instrumentais devem ser entendidas como a passagem para um novo paradigma.

Quando o LAMO3d, (Laboratório de Modelos 3d e Fabricação Digital - PROURB FAU-UFRJ) adquire as primeiras máquinas de Fabricação Digital, a necessidade de instrumentalização em desenho paramétrico, tendo como objetivo final a fabricação digital, pareceria ser a solução de todos os problemas para enfrentar a inovadora arquitetura contemporânea. Contudo, depois de um tempo percebemos num "flashback" que esta discussão relembra a que existiu há cerca de 30 anos atrás quando o desenho assistido por computador (CAM) foi introduzido na prática profissional e na academia. Mas tal como então a simples utilização de ferramentas tecnologicamente mais evoluídas não significa que a disciplina tenha absorvido a especificidade da nova tecnologia. No nível seguinte a possibilidade de automatizar o projeto para que possa responder às solicitações do meio, também não significa por si um melhor projeto. A exploração de novos métodos que promovam a qualidade, a justiça social ou pensamento de vanguarda necessitam, além da 
instrumentalização, de uma revisão metodológica do ato de projetar que faz o oficio.

Os novos processos digitais permitem explorar soluções paramétricas ultrapassando uma lógica sequencial problema> projeto> construção com uma solução única. O projeto paramétrico pode permitir ainda obter famílias de soluções utilizando novas lógicas de modelação e modificando a sequência de projeto.

Partindo destas premissas, surge a incerteza de como instrumentalizar o desenho paramétrico visando a fabricação digital e superando o discurso metódico ferramental.

Utilizar o CAD para "passar a limpo" o desenho feito a mão, não foi considerado uma mudança de paradigma, tal como não há mudança de paradigma quando substituímos o estilete pela máquina de corte a laser. Para haver mudança de paradigma é necessário incorporar um discurso que legitime as intervenções instrumentais. Enquanto não tivermos esse discurso legitimador estaremos, como a inicios do século XX, desenhando carruagens puxados por cavalos, ao invés de carros.

A procura de um discurso ou de uma narrativa legitimadora do produto é sem dúvida melhor do que opção da técnica pela técnica. Só este entendimento nos permite perceber a mudança que os novos processos e ferramentas poderão trazer para o projeto, expandindo as possibilidades anteriores.

Para suprir esta lacuna foi proposto e realizado o Workshop chamado de Abrigos Sensíveis que atua na direção da superação do aprendizado que tem como base unicamente a instrumentalização em fabricação digital. Neste sentido há um esforço em superar a fase de pura instrumentalização, e induzir a procura de um método conceitual.

\section{Procedimentos Metodológicos}

O Workshop se transformou em uma atividade anual conjunta entre três laboratórios da UFRJ, LAMO, LAURD e NANO, e pretende incorporar contributos de áreas diferentes como da arquitetura, das belas-artes e das ciências. Estas diferentes áreas estão explorando as novas possibilidades do digital para integrar na sua prática. Tendo estas áreas diferentes especificidades, encontram diferentes soluções de como incorporar o digital, o que torna fundamental a partilha de experiências e processos. Ao incluir estas diferentes áreas, pretende-se incluir diferentes modus operandi sobre a utilização do digital redefinindo os processos de pesquisa em projeto. Relativamente ao método tradicional este tipo de ferramentas e processos permite intervir em todas as fases de projeto numa sequência assincrónica ao contrário de alguns métodos anteriores que devido à dificuldade do próprio médium favoreciam um projeto sequencial e unidirecional (Duarte/Celani e Oxman 2011).

Esta iniciativa é destinada a participantes com menor experiência nos processos digitais de projeto. Como tal é necessário introduzir o ensino de projeto assistido por computador, desenho associativo e paramétrico, fabricação digital e de novas mídias como o Arduino. O Arduíno tem permitido aos alunos e às universidades o acesso a mecanismos responsivos de maneira acessível, que lhes permite antever possibilidades de conceber mecanismos que tornem os abrigos em estruturas responsivas em tempo real. A instrumentalização destes processos incluiu a modelação digital com o Rhinoceros, a iniciação na programação visual utilizando o aplicativo Grasshopper, incluindo diversos plugins para a geração de múltiplas alternativas de projeto. É explicada a vantagem da integração digital destes programas com a manufatura assistida por computador com a capacitação em corte a laser, capacitação em impressão 3d, capacitação em técnicas de marcenaria serralharia e montagem de produto. Também é feita a capacitação em robótica responsiva via Arduíno. Ou seja o projeto é encarado dentro de um contínuo digital, que também influencia o próprio projeto e a sequência de operações utilizada. No momento em que uma linha no desenho deixa de ser apenas uma representação, mas passa a ser também uma linha de corte numa operação posterior o processo integrado adquire um novo sentido.

Contudo o ponto de partida do projeto é de índole conceitual. O exercício sugere determinados mecanismos responsivos primários e pretende-se que num período alargado mas intenso os alunos concebam, fabriquem, montem a solução. Esta solução deve incluir os mecanismos que permitem o movimento, o que só é possível com a fabricação e testando as soluções com modelos físicos. Assim o ensino destas diferentes áreas, promovendo não só a reflexão como a aprendizagem e uso destes novos processos e ferramentas mas definindo um conceito. Assim mais do que um instrumento o processo permite múltiplas propostas, em que a tentativa e erro permitem aperfeiçoar a solução dando ao aluno um aprendizado valioso, com base na prática. Este aprendizado poderá ser utilizado futuramente para o aluno conceber, cortar e manufaturar os seus próprios projetos. Utilizando apenas métodos analógicos, quem concebe, produz e constrói o projeto raramente é a mesma pessoa, dificuldade que pode ser ultrapassada com recurso à integração digital (Branko Kolarevic).

\section{Resultados}

\section{Do formato e procedimentos}

A Oficina é do tipo imersão, começa num sábado e acaba no outro domingo totalizando 9 dias de intensa atividade. Sempre realizada em época de férias ou aproveitando a greve. Para os 4 primeiros dias propomos palestras na parte da manhã e tutoriais na parte da tarde.

A proposta é a de trabalhar entre 4 a 5 grupos, cada grupo acaba instalado em uma das generosas mesas de Peroba do Campo do LAMO3d. Este escopo nos deixa aproximadamente com um máximo de 35 alunos atendidos. Tivemos ainda 4 professores como participantes, 3 deles somente na fase de tutoriais. O LAMO possui uns 15 
monitores a nível de graduação e alguns simpatizantes a nível de mestrado e doutorado que além de colaborar no cotidiano do WS, acompanham as palestras com atenção e os tutoriais com dedicação.

São eles de fundamental importância porque geralmente com a sua experiência colaboram na execução da fabricação digital, eles dominam a nível básico os materiais e as técnicas de corte a laser, de impressão $3 d$, de router cnc e ferramental de oficina. Pelo menos um deles fica ancorado em cada grupo, sendo que os que mais dominam o desenho paramétrico ficam dando ajudas pontuais.

A temática está pouco dominada por professores acadêmicos com doutorado. Existe uma defasagem, pelo menos nas universidades públicas, entre o doutor e o tempo em que começa a dar as suas aulas e realizar as suas pesquisas. Neste sentido poucos doutores dominam a temática a nível de softwares, e principalmente de processos de fabricação. Então surge a necessidade de realizar os tutoriais de instrumentalização por profissionais que geralmente não possuem titulação acadêmica, o que resulta geralmente em desqualificação da equipe por parte das instituições de financiamento, CAPES, CNPQ e no nosso caso FAPERJ. Difícil explicar para eles o por quê de 9 diárias e uma passagem nacional para um garoto de 22 anos que mal acabou de se formar!

$\mathrm{Na}$ parte de desenho paramétrico, tivemos 3 tutores, Victor Sardenberg, Verônica Natividade e Orion Campos. cada um deu uma palestra, e todos eles deram tutoriais. Sendo as duas primeiras tardes dedicadas à introdução Rhinoceros+Grasshopper.

Na parte de eletrônica tivemos também 2 tutores, Lucas de Sordi e Marlus Araujo, cada um dando a sua palestra e tutorias de Arduino com sensores, e principalmente com motores para chegar nas propostas responsivas.

Na parte de computação e integração digital tivemos um palestrante tutor, Gonçalo Castro Henriques que partilhou experiências anteriores, somado ao trabalho dos monitores. Finalmente nomes de peso acadêmico realizam palestras

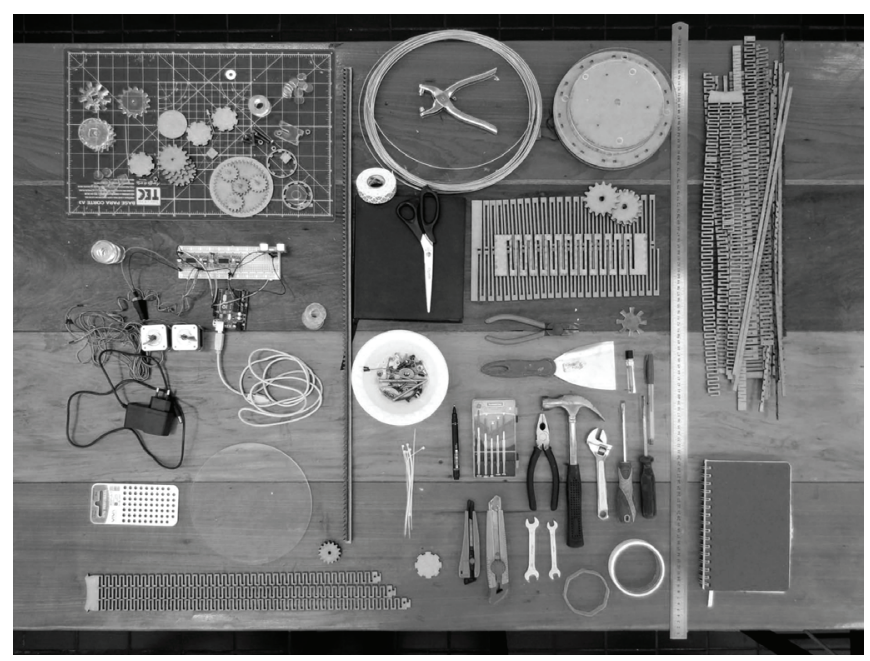

Figura 1: Ferramentas e mecanismos do workshop abrigos sensíveis. pontuais, Andrés Passaro, Arthur Lara, Guto Nóbrega.

De maneira genérica os alunos foram instrumentalizados nas técnicas de desenho paramétrico e fabricação digital, sendo capacitados também no corte a laser e impressão $3 d$. Inicialmente foram propostos 5 mecanismos primários (cortina; polvo; concha; lanterna chinesa e flor brinco de princesa), a partir dos quais forma desenvolvidos 5 projetos responsivos. A partir do quarto dia cobrávamos apresentações diárias sobre conceitos, ideias, mecanismos e forma, e essa cobrança foi fundamental para o correto desenvolvimento dos projetos. O resultado incorporou o aprendizado e a tentativa e erro do WS para conseguir resultados que superaram a dicotomia forma - instrumentalização. O rigor do discurso cobrado durante as apresentações diárias do projeto superou o encanto apelativo apenas da técnica e dos instrumentos. Se procura uma superação que transborda o aprendizado em novos instrumentos, não se trata de tentar fazer o que fazíamos antes somente utilizando uma nova instrumentação e sim de procurar novas possibilidades a serem executadas com estes novos instrumentos.

\section{Desafio do workshop. Conceito arte e técnica.}

O workshop desenvolveu 5 propostas de abrigos sensíveis. Procurando criar abrigos que incorporassem no seu conceito; ou a forma de interagir, ou o mecanismo de atuação ou a própria geometria que nasce da síntese de projeto.

A ideia base para ultrapassar o básico instrumental foi estimular uma ação integrada nos vários momentos do processo criativo, evitando assim o pensamento sequencial, da ideia para a forma que vive com o vai-e-vem da forma para a ideia. Um mecanismo que está para além da retórica, porque tem de ser construído e ao mesmo tempo funcionar, dentro de um design que é encarnado através da matéria. $O$ projeto ganha escala, movimento, ao mesmo tempo em que procura uma narrativa própria, incorporando o maquínico o sensorial e o algoritmo no cerne do processo. Na ação projetual é incorporada a fabricação digital (CAM) que vai até às engrenagens dos mecanismos antes simulados (CAE) e a concepção de projeto(CAAD). Um processo de simultaneidade entre parte e o todo. A parte são células que formam tecidos de um abrigo que apenas antevê o exoesqueleto; imprescindível para o abrigo e o seu mecanismo ganharem vida própria. Para este processo é necessário conhecimento e prática, a teoria e o confronto empírico de construir testar e voltar a tentar novamente.

O projeto foi concebido por algoritmos através de programação visual (grasshopper) pensando nas interfaces sensoriais que ativam os movimentos. Os sinais chegam através de sensores diversos a uma interface eletrônica, no caso o Arduino, que é encarregado de transmitir os dados ao computador onde está o algoritmo. $\mathrm{O}$ algoritmo aqui é tratado como um mecanismo mais brando "soft" (não software) que controla a relação entre as geometrias e o seu devir, ao mesmo tempo que transmite as ações ao Arduino. A partir deste momento o Arduino relata de maneira mais vigorosa "hard" as 
instruções e ativam os motores que giram rodas dentadas. Em um primeiro momento a geometria é realizada no computador e as simulações são ativadas com o mouse. Em um segundo momento se proíbe o mouse, e as simulações no computador são ativadas pelos sensores plugados no Arduino, finalmente se proíbe o computador, e já não mais se trata de simulações e sim de um produto responsivo.

O Workshop começa com uma imersão intensa entre teoria e prática, iniciado por palestras que abordam projetos físicos e possibilidades construídas, mostrando sempre a sua viabilidade projetual. As palestras em geral assumem o ponto de vista do maker, físico e tecnológico, de geração, de fabricação, a simulação a interação e o entendimento de um ambiente sensível e responsivo. A partir daqui cada grupo procura apontar referências sólidas das suas experiências, e assim o entusiasmo dos participantes cresce, mesmo para aqueles que nunca dominaram nem o computador nem o alicate. Não se trata só de novos instrumentos. Das ideias surgem formas que se reconfiguram num processo empírico de tentativa e erro.

Os tutores buscam simbioses entre os processos físicos e digitais, trabalham e conversam em conjunto para orientar os gruposdetrabalho. Osgrupostrabalhamarduamente, reuniões e pontos de situações, sugestões, tentativas, e os projetos começam a se materializar, e as facilidades em fabricação aumentam a velocidade de criação vertiginosamente.
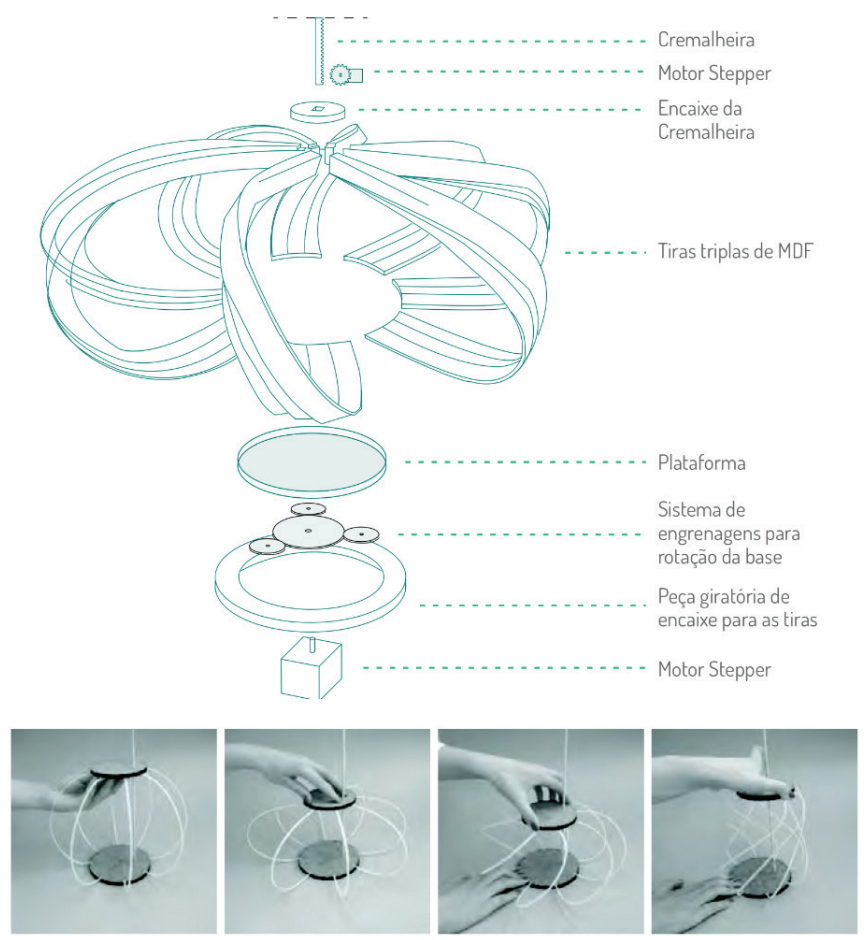

0 abrigo realiza dois movimentos independentes: move-se no eixo vertical em ambos os sentidos, assim a reação das tiras simula o movimento de respiração, e o segundo movimento é

\section{5 conceitos 5 mecanismos}

Descrever o conceito dos 5 mecanismos propostos é falar da estratégia deste workshop. Foram propostos 5 movimentos a serem apropriados pelos grupos, estes movimentos foram designados como: cortina, polvo, concha, lanterna chinesa, flor brinco-de-princesa, nomes que foram associados diagramas de movimentos. $O$ dado de partida era assim um nome e um diagrama. E o resultado uma interação entre o físico e digital que podia extrapolar o diagrama original.

G1 - A ÉFIRA Parte de um mecanismo com um eixo vertical em que os dois topos giram em sentido contrário. É um tipo de movimento que cria uma torção em uma pele exterior estriada ligando os dois topos. Este movimento em espiral é ativado através de um sensor de presença para o Arduino o qual transmite ao algoritmo que controla o movimento e devolve o sinal. O Arduino faz girar um motor que transmite aos dois topos no eixo vertical uma movimentação que entre eles é inversa, a qual é transmitida por rodas dentadas que torcem a estrutura, em um sentido ou em outro de acordo com a presença do usuário.

G2 - O ORIGAMI é um movimento de expansão simultânea que parte desde um centro em direção a 4 arestas de uma mesma face de um cubo transparente. Os pontos suspendem por ora uma superfície dobrada/contraída, que se desdobra/expande na presença humana a qual é detectada por um potenciómetro (leap motion). O potenciômetro transmite os dados ao Arduino, passa ao algoritmo e retorna o sinal ao Arduino, que finalmente transmite a informação a 4 motores que produzem os movimentos por cabos e roldanas,
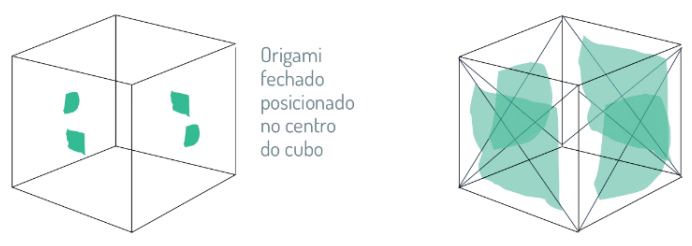

Drigami aberto fechando o espaço etransformando-o em abrigo
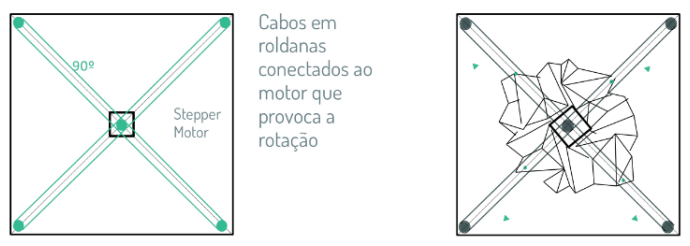

As dobras do origam possibilitam sua abertura ou dechendento, direção de rotaçāo do motor

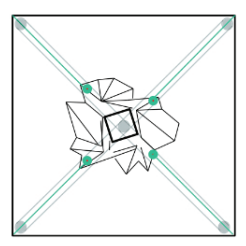

Pontos da conexão da rodana com os cabos que irão correr e puxar o origami, possibilitando sua abertura ou fechamento

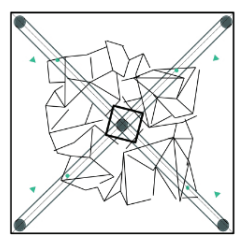

0 ângulo de $90^{\circ}$ estabelece 0 máximo d rotação necessária para abrir ou fechar 0 origami completamente

Figura 2: Desenvolvimento da Èfira.

Figura 3: Desenvolvimento do Origami. 
resultando em movimentos de dobras e desdobras.

G3 - XTENSÃO surge da tensão entre cabos e barras, o que resulta em uma estrutura tensionada em módulos dentro do princípio do «tensigrity». 5 pares de barras e cabos agrupamse numa disposição circular, as barras de cada módulo ficam na vertical e são dispostas num arranjo circular (polar array). O movimento de expansão retração das barras no sentido do centro da circunferência faz com que toda a estrutura se reconfigure. Movimentos humanos e impulsos vegetais atuam a estrutura que passa ao Arduino e ao algoritmo, este devolve a um conjuntos de 1 motor rotatório no cento e 5 roldanas nas pontas da circunferência. $O$ motor transmite o movimento às roldanas que contraem ou retraem as barras em função do centro. A rotação faz as barras de cada modulo se aproximar ou distanciar do centro, e com elas os cabos de cada modulo reconfiguram-se mudando a forma da estrutura. Parando o estímulo tanto as barras como a estrutura, regressam ao ponto inicial de equilíbrio.
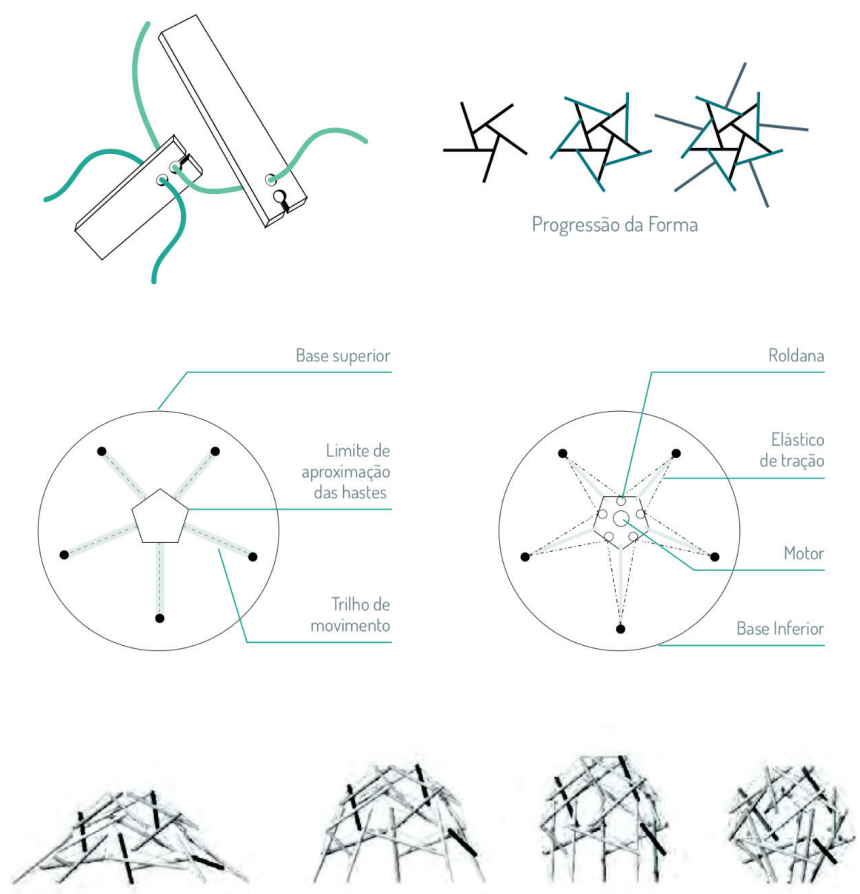

Figura 4: Desenvolvimento da Xtensão.

G4 - O CATERPILLAR é constituído por faixas longitudinais paralelas ao solo, que são sensíveis à aproximação humana e curvam-se como uma concha para receber o visitante. As tiras se movimentam de acordo com a distância do estímulo. São tensionadas ou contraídas a partir dos extremos e vão ficando progressivamente arqueadas. Um sensor transmite a aproximação e é passado um sinal ao algoritmo via Arduino. $\mathrm{O}$ algoritmo transmite os dados ao Arduino que controla progressivamente quatro motores de fuso no sentido das tiras. A aproximação humana vai ativando progressivamente cada um dos motores, e vai desativando em sentido contrario a medida que se distancia.

Figura 5: Desenvolvimento do Caterpillar.

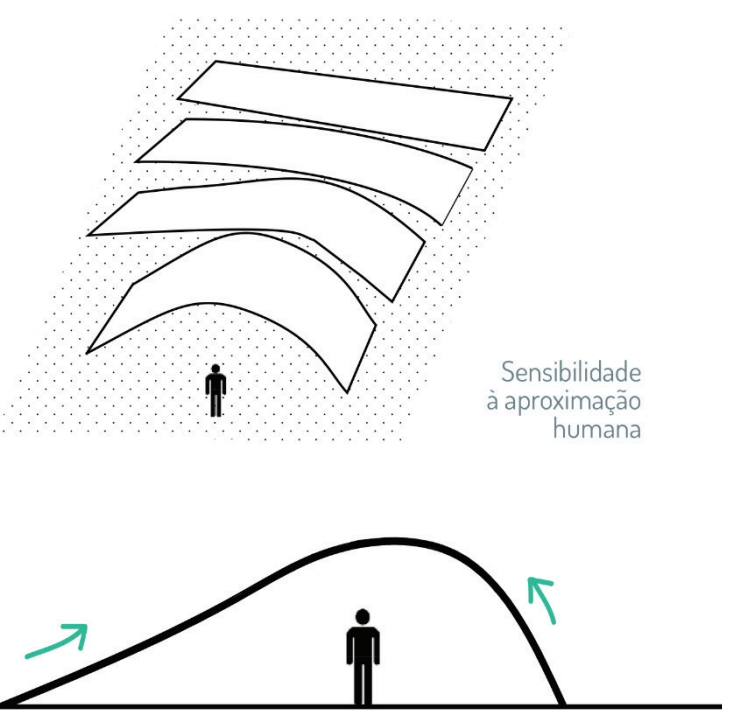

Movimento desliza com um raio oblíquio ao piso
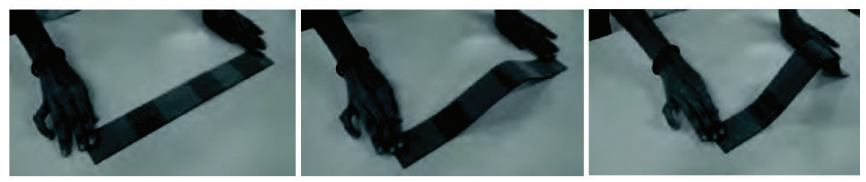

G5 - O MUSCIPULLUS se apresenta como uma planta carnívora, este abrigo aguarda o visitante e se abre na sua chegada. A forma utiliza um conjunto de eixos verticais que estão arranjados à volta de um círculo (array polar). Cada um destes eixos está associado a um painel. E um movimento sincronizado dos 5 painéis a planta carnívora abre-se para a passagem humana e se fecha como uma armadilha quando alguém entra. A interface é análoga aos anteriores sensor Arduino, algoritmo. $O$ algoritmo transmite ao Arduino que passa o sinal a um motor rotativo central que através de alavancas passa o movimento aos cinco eixos. Assim a planta carnívora abre-se e fecha-se interagindo com o visitante. 


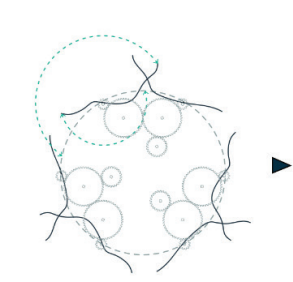
1. Portas Abertas e
Movimentaçāo das Portas

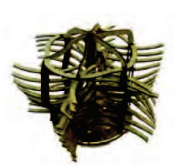
através das Engrenagens

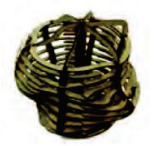

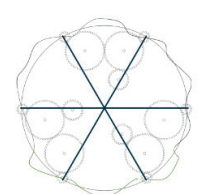

Pórtico de Sustentaçāo das Portas

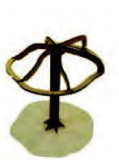

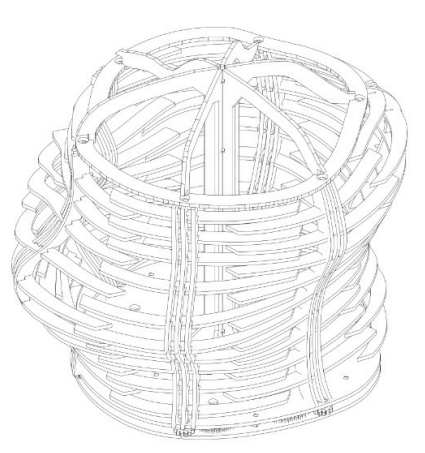

COMPONENTES

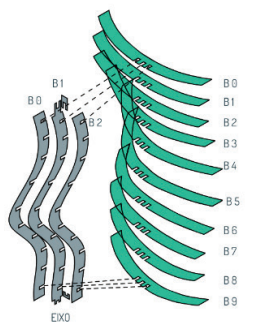

Figura 6: Desenvolvimento do Muscipullus.

A rasgos gerais esta formatação procurou resgatar mecanismos conceituais aplicados ao projeto, integrou processos e tecnologias ligadas à ação e ao construir. Falando de linguagem, a retórica é mais difícil e somente consegue fugir da tecnologia se for dominando essa própria tecnologia. Assim em vez de instrumentalizar em novas ferramentas vislumbramos novas posturas de mimeses que apontam para um discurso de bioforma, de morfogénesis, com base na proto-mecânica, algorítmica e sensorial. O Workshop Abrigos Sensíveis é só o começo desta nova discussão.

\section{Conclusões}

A discussão acima oferece o entendimento que por si só as disciplinas de instrumentalização e os novos processos não nos levam de maneira autônoma a mudanças significativas dentro do projeto. Desta maneira todo o Workshop girou em torno da necessidade de encontrar uma narrativa própria, que não seja literal e que tenha a solidez suficiente para efetivar/ legitimar uma iminente mudança de paradigma.

Os resultados "interessantes" acima descritos, por si só, não introduzem mudanças suficientes para justificar qualquer mudança de paradigma. No entanto a solidez da proposta consegue ultrapassar mais um "passar a limpo" que não traria grandes consequências além de jogos formais hedonistas.

Neste sentido os resultados além de questionar o processo tradicional via aprendizagem de novas ferramentas, procura a fundamentação destes novos processos, invadindo novas áreas do conhecimento. Este aprendizado combate o conhecimento especializado e fracionado, sendo testadas soluções na prática através da sua construção integrando processos digitais e analógicos, num processo continuo.

A modo de desafio, podemos definir que o trabalho está "ainda em andamento" o que nos proporciona uma janela aberta para exploração de "outros" modos, outras maneiras de produção. No LAMO3d, a máquina de corte a laser foi o primeiro passo para deixar de lado o estilete, porém é necessário entender que utiliza-la como estilete é um erro conceitual. A janela aberta vai neste sentido, o de perceber um caminho distante, onde possamos superar entraves, e aproveitar ao máximo o ferramental tecnológico, para abordar o processo de uma outra maneira. O WS conseguiu produzir uma massa crítica nesta direção, já que conseguimos discutir questões sobre o desenho paramétrico, com os Tutoriais de Victor Sardenberg, Orion Campos e Verônica Natividade; passamos pela robótica com os tutoriais de Lucas de Sordi; abordamos a impressão 3d e prototipagem rápida com Marlus Araujo; ainda abordamos o tema de hibridizações via interatividade de organismos vivos com Guto Nóbrega coordenador do NANO. Também começamos a observar construções efêmeras para situações extremas com Arthur Lara, e vivenciamos processos de computação e fabricação digital com Gonçalo Castro Henriques quem deixou uma direção neste sentido para o nosso próximo WS.

A janela é uma rede que vai sendo costurada pouco a pouco, adquirindo tecnologia equipamentos, conhecimento e muita vontade do fazer. Esta soma de acontecimentos abre a janela um pouco ao modo do "Anjo da Historia", de (Benjamim 2015) onde começamos a perceber, a medida que olhamos para trás, amontoados de modos de produção que estão sendo superados. Claro que sempre desde um ponto de vista menos nihilista que o de Benjamim, no caso o nosso Anjo estaria virado para a frente, não mais com a boca desencaixada e sim com um sorriso de encanto.

\section{Referências}

Abrigos Sensíveis. (2015) Rio de Janeiro, RioBooks - Prelo. Passaro Andrés, Duque Rebeca, Rohde Clarice, Vianna Elisa. Benjamin, Walter. (2015) O anjo da historia. Belo Horizonte. Editora Autêntica

Duarte, José Pinto. Celani, Gabriela. PUPO, Regiane. (2011) Inserting computational technologies in architectural curricula. In Gu, Ning and Wang, Xiangyu (ed.), Computational Design Methods and Technologies: Applications in CAD, CAM and CAE Education, Hershey, PA: IGI Global.

Henriques, Gonçalo Castro. Bueno, Ernesto. (2009) Geometrias Complexas e Desenho Paramétrico. In: Vitruvius / Drops, n.30. Romano Guerra·2010, Novembro 2009.

Kolarevic, Branko, Designing and Manufacturing Architecture in the Digital Age, (2003) Spoon Press Taylor \& Francis Group, Nova Iorque.

Moneo, Rafael. (1999) Los 90 entre la compacidad y la fragmentación. In Arquitectura Viva, $n^{\circ}$ 66. Madrid, mayo- 
junio, p 17-24.

Oxman, Rivka, (2006) Theory and Design in the First Digital Age", em Design Studies, Vol. 27 No. 3.

Passaro, Andrés. (2008) A Forma Ausente". In Revista NOZ \#2. PUC-Rio, Rio de Janeiro.

Quetglas, Josep. En tiempos irregulares. Revista El Croquis $n^{\circ}$ 106/107 - Arquitectura Española. Madrid, 2002.

Imagens:

Figura 1: Laura Lago

Figura 2 a 6: Abrigos Sensíveis
WorkShop Abrigos Sensíveis - Ficha técnica:

Coordenadores: Andrés Passaro, Guto Nóbrega. Organização: Clarice Rohde, Elisa Vianna, Gonçalo Castro Henriques, Laura Basile, Rebeca Duque Estrada. Tutores: Arthur Lara, Lucas de Sordi, Marlus Araújo, Oríon Campos, Verônica Natividade, Victor Sardenberg. Comité Científico: Carlos Feferman, Luiz Felipe Cunha, Malú Fragoso, Naylor Vilas Boas, Rodrigo Cury. Monitores e Fotógrafos: Diogo de la Vega, Felipe Madeira, Filipi Dias, João Magnus, Jonas Abreu. 